\title{
Comparison of standard-dose and half-dose sorafenib therapy on clinical outcome in patients with unresectable hepatocellular carcinoma in field practice: A propensity score matching analysis
}

\author{
HIROKI NISHIKAWA $^{1 *}$, YUKIO OSAKI ${ }^{1}$, MASATSUGU ENDO ${ }^{1}$, HARUHIKO TAKEDA $^{1 *}$, \\ KAORU TSUCHIYA ${ }^{2}$, KOUJI JOKO ${ }^{3}$, CHIKARA OGAWA $^{4}$, HIROYOSHI TANIGUCHI ${ }^{5}$, \\ ETSURO ORITO $^{6}$, YASUSHI UCHIDA ${ }^{7}$ and NAMIKI IZUMI ${ }^{2}$; \\ JAPANESE RED CROSS LIVER STUDY GROUP
}

\author{
${ }^{1}$ Department of Gastroenterology and Hepatology, Osaka Red Cross Hospital, Tennoji-ku, Osaka 543-8555; \\ ${ }^{2}$ Department of Gastroenterology and Hepatology, Musashino Red Cross Hospital, Musashino, Tokyo 180-8610; \\ ${ }^{3}$ Center for Liver-Biliary-Pancreatic Diseases, Matsuyama Red Cross Hospital, Matsuyama 790-8524; \\ ${ }^{4}$ Department of Gastroenterology and Hepatology, Takamatsu Red Cross Hospital, Takamatsu 760-0017; \\ ${ }^{5}$ Department of Gastroenterology, Japanese Red Cross Medical Center, Shibuya-ku, Tokyo 150-8935; \\ ${ }^{6}$ Department of Gastroenterology and Hepatology, Nagoya Daini Red Cross Hospital, Showa-ku, Nagoya 486-8650; \\ ${ }^{7}$ Department of Gastroenterology, Matsue Red Cross Hospital, Matsue 690-8506, Japan
}

Received July 9, 2014; Accepted August 26, 2014

DOI: 10.3892/ijo.2014.2654

\begin{abstract}
The aims of the present study were to examine whether unresectable hepatocellular carcinoma (HCC) patients treated with initial dose of sorafenib of $400 \mathrm{mg} /$ day (half-dose group) had comparable treatment efficacy, safety and survival merit as compared with those treated with initial dose of sorafenib of $800 \mathrm{mg} /$ day (standard-dose group) in a multicenter large study. For reducing the bias in patient selection, we compared clinical outcomes of these two groups using propensity score matching analysis. A total of 465 patients were treated with sorafenib at fourteen hospitals in Japanese Red Cross Liver Study Group from 2008 to 2013. After propensity score matching, 139 matched HCC patients were selected for analysis in both groups. We retrospectively compared overall survival (OS), progression-free survival (PFS), best treatment response and sorafenib related serious adverse events (SAEs) in the two groups. There were no relevant differences in terms of OS (median OS intervals: 9.2 months in the standard-dose group and 9.7 months in the half-dose group, $\mathrm{P}=0.350$ ), PFS (median PFS intervals: 3.4 months in the standard-dose group
\end{abstract}

Correspondence to: Dr Hiroki Nishikawa, Department of Gastroenterology and Hepatology, Osaka Red Cross Hospital, 5-30 Fudegasaki-cho, Tennoji-ku, Osaka 543-8555, Japan

E-mail: h-nishikawa@osaka-med.jrc.or.jp

${ }^{*}$ Contributed equally

Key words: hepatocellular carcinoma, sorafenib, standard dose, half dose, clinical outcomes and 3.2 months in the half-dose group, $\mathrm{P}=0.729$ ) and best treatment efficacy (objective response rate: $\mathrm{P}=0.416$; disease control rate: $\mathrm{P}=0.719)$. Grade 3 or more SAEs were observed in 37 patients $(26.6 \%)$ in the standard-dose group and 33 patients $(23.7 \%)$ in the half-dose group $(\mathrm{P}=0.580)$. Furthermore, in all subgroup analyses according to Child-Pugh classification and Barcelona Clinic Liver Cancer stage, there were no significant differences in the two groups. In conclusion, unresectable HCC patients treated with initial half-dose sorafenib had comparable prognosis compared with those treated with initial standard-dose sorafenib.

\section{Introduction}

Hepatocellular carcinoma (HCC) is one of the most common causes of cancer-related mortality worldwide in terms of incidence with 626,000 new cases per year, accounting for $5.7 \%$ of all new cancer cases (1-5). Annual incidence rates of HCC are highest in Sub-Saharan Africa and East Asia, where $~ 85 \%$ of all cases occur (1-5). The vast majority of HCC cases occur in the context of hepatitis virus or alcohol related chronic liver disease and consequently many patients with HCC present with liver dysfunction and experience a high rate of comorbidity (1-5). Thus, HCC is a heterogeneous disease with regard to etiology as well as clinical presentation, presenting challenges for disease management. The therapies of HCC have significantly changed in the last few decades $(1,2,5)$. With these changes and advances in medical technology such as diagnostic imaging and surveillance programs for detecting earlier stage $\mathrm{HCC}$, survival in patients with $\mathrm{HCC}$ has markedly improved $(4,5)$. However, unfortunately, $<20 \%$ of HCC patients are amenable to curative therapy such as liver transplantation, surgical resection or ablative therapies. Furthermore, HCC 
often recurs even after curative therapy and survival in HCC patients with advanced stage remains poor $(5,6)$.

Sorafenib is a multi-kinase inhibitor that blocks tumor growth and cell proliferation $(7,8)$. Although systemic chemotherapy such as doxorubicin was not demonstrated to be effective for the treatment of advanced HCC for several decades, two randomized phase III studies, namely the Sorafenib HCC Assessment Randomised Protocol (SHARP) study and the Asian Pacific study, showed that sorafenib therapy obtained survival benefit over placebo group for patients with unresectable HCC, and molecular targeted therapy with sorafenib is currently approved for use as first-line systemic chemotherapy in these patients $(7,8)$. Furthermore, results with regard to the treatment efficacy and safety of sorafenib therapy for HCC in clinical practice were demonstrated by several field practice experiences, including the SOraFenib Italian Assessment (SOFIA) study and the Global Investigation of therapeutic DEcisions in HCC and Of its treatment with sorafeNib (GIDEON) study (9-12). However, to the best of our knowledge, few studies compared the clinical outcomes and safety between different initial doses of sorafenib administered to HCC patients $(10,13)$. In Japan, the investigations for optimal initial dose of sorafenib for unresectable HCC are still underway, although two pivotal studies recommended initial dose of sorafenib of $800 \mathrm{mg} /$ day $(7,8)$.

The aims of the present study were thus to examine whether HCC patients treated with initial dose of sorafenib of $400 \mathrm{mg} /$ day (half-dose) had comparable treatment efficacy, safety and survival merit as compared with those treated with initial dose of sorafenib of $800 \mathrm{mg} / \mathrm{day}$ (standard-dose) in a multicenter large study. For reducing the bias in patient selection, we compared clinical outcomes of these two groups using propensity score matching analysis.

\section{Patients and methods}

Patients. A total of 465 consecutive HCC patients have been treated with sorafenib at fourteen hospitals in Japanese Red Cross Liver Study Group from January 2008 to July 2013. Sorafenib therapy was indicated in patients with unresectable HCC determined by dynamic computed tomography (CT); i) existence of extrahepatic metastases; or ii) refractory to previous HCC therapies such as transcatheter arterial chemoembolization (TACE); or iii) unsuitability for TACE for anatomical reasons; or iv) vascular invasion such as tumor thrombus in the portal vein (14-16). Patients with a performance status (PS) of 3 or 4 according to the Eastern Cooperative Oncology Group (ECOG) classification were excluded $(14,15)$. Since the aim of the current analysis was to compare clinical outcomes of HCC patients treated with initial dose of sorafenib of $800 \mathrm{mg} /$ day and those treated with initial dose of sorafenib of $400 \mathrm{mg} /$ day, patients treated with initial dose of sorafenib of 600 or $200 \mathrm{mg} / \mathrm{day}(\mathrm{n}=22)$ were excluded from the current analysis. Patients with Barcelona Clinic Liver Cancer (BCLC)-A or D $(n=6)$ were also excluded. Thus, a total of 437 patients $(n=184$ in the standard-dose group and $\mathrm{n}=254$ in the half-dose group) were analysed in the present study (Fig. 1). We retrospectively compared overall survival (OS), progression-free survival (PFS), treatment response and sorafenib related adverse events in the two groups.
The present study comprised a retrospective analysis of patient records and all treatments were conducted in an open-label manner. The ethics committees of all facilities that participated in this study approved the present study protocol and this study protocol complied with all of the provisions of the Declaration of Helsinki.

HCC diagnosis and sorafenib therapy. HCC was diagnosed as described previously $(14,15)$. Briefly, dynamic CT or magnetic resonance imaging of the liver was undertaken prior to sorafenib therapy in all analysed patients. In some patients who presented with atypical liver tumors, we conducted ultrasound-guided tumor biopsy. HCC was diagnosed by radiological or histological method according to European Association for the Study of Liver guideline (17).

As for initial dose of sorafenib, for patients with no risk factors, we introduced the recommended initial dose $400 \mathrm{mg}$ twice a day of sorafenib $(800 \mathrm{mg} /$ day $)$. The initial dose was reduced according to factors such as body weight, age, ECOG-PS and liver function. During sorafenib treatment, each attending physician decided to reduce daily dose of sorafenib according to the grades of adverse events or ECOG-PS. In patients treated with half-dose sorafenib with good tolerance, dose escalation of sorafenib was allowed. Temporary interruption was maintained until the symptoms resolved to grade 1 or 2. We assessed the treatment response of sorafenib every 4-8 weeks after the initiation of sorafenib therapy by modified Response Evaluation Criteria in Solid Tumors (mRECIST) and/or tumor markers $(14,15,18,19)$. Sorafenib therapy continued until disease progression, unacceptable drug-related toxicity, or the patient's wish to discontinue treatment. After discontinuation of sorafenib therapy for any reason, any other therapies such as TACE or systemic chemotherapy were permitted according to the tumor status or the general status of each patient $(14,15)$.

Evaluation of treatment efficacy. Best treatment efficacy of sorafenib during treatment was assessed in accordance with the mRECIST criteria and/or tumor marker levels as mentioned above $(14,15,18)$. The treatment efficacy was classified as: i) complete response (CR), ii) partial response (PR), iii) stable disease (SD) and progressive disease (PD). CR was defined as disappearance of any arterial enhancement within all target tumors. PR was defined as $\geq 30 \%$ decrease in tumor size as determined by evaluation of the sum of the diameters of the target tumors, whose size was estimated using unidirectional measurement. PD was defined as $\geq 20 \%$ increase in tumor size as determined by evaluation of the sum of the maximal dimensions of the target tumors. SD was defined as the absence of either PR or PD $(15,18)$. The objective response rate (ORR) was defined as the percentage of patients who had a best response rate of CR and PR. The disease control rate (DCR) was defined as the percentage of patients who had a best response rate of CR, PR and SD.

Safety evaluation of sorafenib therapy. Sorafenib related toxicities, including hand foot skin reaction (HFSR), rash, diarrhea, hypertention, fatigue, liver injury, gastrointestinal bleeding and lung injury were evaluated using Common Terminology Criteria for Adverse Events (CTCAE) version 3.0. 


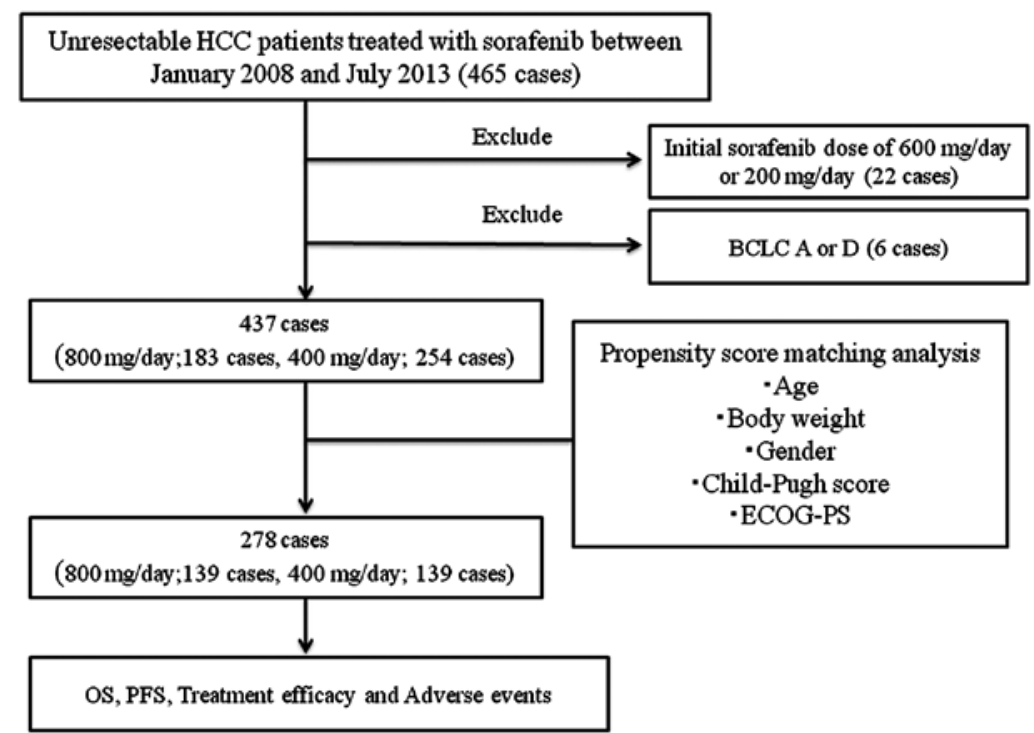

Figure 1. Study design. HCC, hepatocellular carcinoma; BCLC, Barcelona Clinic Liver Cancer; ECOG-PS, Eastern Cooperative Oncology Group Performance Status; OS, overall survival; PFS, progression-free survival.

Table I. Baseline characteristics between patients treated with initial dose of sorafenib of $800 \mathrm{mg} /$ day (standard-dose group) and those treated with initial dose of sorafenib of $400 \mathrm{mg} /$ day (half-dose group) before propensity score matching.

\begin{tabular}{lccr}
\hline & $\begin{array}{c}\text { Standard-dose group } \\
(800 \mathrm{mg} / \text { day, } \mathrm{N}=183)\end{array}$ & $\begin{array}{c}\text { Half-dose group } \\
(400 \mathrm{mg} / \text { day, } \mathrm{N}=254)\end{array}$ & P-value \\
\hline Age (years) & $67.2 \pm 9.9$ & $71.7 \pm 10.2$ & $<0.001^{\mathrm{a}}$ \\
Gender, male/female & $156 / 27$ & $200 / 54$ & $0.104^{\mathrm{b}}$ \\
Height (cm) & $162.4 \pm 8.2$ & $159.2 \pm 16.7$ & $0.020^{\mathrm{a}}$ \\
Body weight (kg) & $60.8 \pm 12.1$ & $57.8 \pm 12.1$ & $0.012^{\mathrm{a}}$ \\
Cause of liver disease & & & \\
B/C/B and C/non-B and non-C & $43 / 96 / 1 / 43$ & $24 / 155 / 4 / 71$ & $0.001^{\mathrm{b}}$ \\
HCC stage, II/III/IV & $4 / 69 / 110$ & $15 / 89 / 150$ & $0.168^{\mathrm{b}}$ \\
ECOG PS, 0/1/2 & $143 / 36 / 4$ & $190 / 55 / 9$ & $0.626 \mathrm{~b}$ \\
Child-Pugh score, 5/6/7/8/9 & $84 / 76 / 21 / 2 / 0$ & $87 / 111 / 32 / 22 / 2$ & $0.002^{\mathrm{b}}$ \\
Serum albumin (g/dl) & $3.59 \pm 0.48$ & $3.49 \pm 0.52$ & $0.043^{\mathrm{a}}$ \\
Total bilirubin (mg/dl) & $0.89 \pm 0.43$ & $0.94 \pm 0.51$ & $0.230^{\mathrm{a}}$ \\
AST (IU/l) & $65.9 \pm 64.9$ & $68.0 \pm 64.1$ & $0.740^{\mathrm{a}}$ \\
ALT (IU/l) & $53.2 \pm 43.1$ & $44.1 \pm 37.6$ & $0.019^{\mathrm{a}}$ \\
Cholinesterase (IU/l) & $176.8 \pm 70.6$ & $157.8 \pm 71.1$ & $0.010^{\mathrm{a}}$ \\
AFP (ng/ml) & $5,491 \pm 18,113$ & $18,973 \pm 108,883$ & $0.054^{\mathrm{a}}$ \\
DCP (mAU/ml) & $11,347 \pm 38,474$ & $23,672 \pm 130,183$ & $0.155^{\mathrm{a}}$ \\
BCLC stage, B/C & $64 / 119$ & $93 / 161$ & $0.762^{\mathrm{b}}$ \\
\hline
\end{tabular}

Data are expressed as number or mean \pm standard deviation. B, hepatitis B virus; C, hepatitis C virus; HCC, hepatocellular carcinoma; ECOG PS, Eastern Cooperative Oncology Group Performance Status; AST, aspartate aminotransferase; ALT, alanine aminotransferase; AFP, $\alpha$-fetoprotein; DCP, des- $\gamma$-carboxy prothrombin; BCLC, Barcelona Clinic Liver Cancer. ${ }^{a}$ Unpaired t-test; ${ }^{b}$ Fisher's exact test.

Statistical analyses. Categorical variables were analyzed by Fisher's exact test. Continuous variables were analyzed by unpaired t-test. OS curves were generated using the KaplanMeier method and compared using the log-rank test. OS was calculated from the initial date of sorafenib therapy until death from any cause or the last follow-up. PFS was calculated from the initial date of sorafenib treatment until the date of progression disease or death from any cause $(14,15)$. Data were analyzed using SPSS (Chicago, IL, USA). Two-tailed probability values of $\mathrm{P}<0.05$ were considered significant. 
Table II. Baseline characteristics between patients treated with initial dose of sorafenib of $800 \mathrm{mg} / \mathrm{day}$ (standard-dose group) and those treated with initial dose of sorafenib of $400 \mathrm{mg} /$ day (half-dose group) after propensity score matching.

\begin{tabular}{|c|c|c|c|}
\hline & $\begin{array}{l}\text { Standard-dose group } \\
(800 \mathrm{mg} / \text { day, } \mathrm{N}=139)\end{array}$ & $\begin{array}{l}\text { Half-dose group } \\
(400 \mathrm{mg} / \text { day, } \mathrm{N}=139)\end{array}$ & P-value \\
\hline Age (years) & $70.0 \pm 8.5$ & $70.1 \pm 9.1$ & $0.903^{\mathrm{a}}$ \\
\hline Gender, male/female & $114 / 25$ & $111 / 28$ & $0.760^{\mathrm{b}}$ \\
\hline Height $(\mathrm{cm})$ & $161.4 \pm 8.3$ & $159.7 \pm 21.0$ & $0.362^{\mathrm{a}}$ \\
\hline Body weight $(\mathrm{kg})$ & $60.1 \pm 12.1$ & $59.1 \pm 14.0$ & $0.528^{\mathrm{a}}$ \\
\hline \multicolumn{4}{|l|}{ Cause of liver disease } \\
\hline $\mathrm{B} / \mathrm{C} / \mathrm{B}$ and $\mathrm{C} /$ non- $\mathrm{B}$ and non- $\mathrm{C}$ & $28 / 76 / 1 / 34$ & $16 / 75 / 2 / 46$ & $0.121^{\mathrm{b}}$ \\
\hline HCC stage, II/III/IV & $4 / 58 / 77$ & $7 / 49 / 83$ & $0.438^{\mathrm{b}}$ \\
\hline ECOG PS, $0 / 1 / 2$ & $107 / 30 / 2$ & $106 / 29 / 4$ & $0.803^{\mathrm{b}}$ \\
\hline Child-Pugh score, 5/6/7/8 & $58 / 60 / 19 / 2$ & $59 / 66 / 11 / 3$ & $0.445^{\mathrm{b}}$ \\
\hline Serum albumin $(\mathrm{g} / \mathrm{dl})$ & $3.56 \pm 0.48$ & $3.59 \pm 0.48$ & $0.616^{\mathrm{a}}$ \\
\hline Total bilirubin $(\mathrm{mg} / \mathrm{dl})$ & $0.88 \pm 0.43$ & $0.91 \pm 0.51$ & $0.551^{\mathrm{a}}$ \\
\hline AST (IU/l) & $58.2 \pm 32.3$ & $65.9 \pm 62.2$ & $0.198^{\mathrm{a}}$ \\
\hline ALT (IU/l) & $46.5 \pm 32.0$ & $44.9 \pm 36.2$ & $0.695^{\mathrm{a}}$ \\
\hline Cholinesterase (IU/l) & $171 \pm 71.6$ & $167 \pm 73.0$ & $0.703^{\mathrm{a}}$ \\
\hline $\operatorname{AFP}(n g / d l)$ & $3,593 \pm 10,551$ & $10,384 \pm 37,885$ & $0.043^{\mathrm{a}}$ \\
\hline $\mathrm{DCP}(\mathrm{mAU} / \mathrm{ml})$ & $8,851 \pm 33,057$ & $20,797 \pm 96,248$ & $0.168^{\mathrm{a}}$ \\
\hline BCLC stage, $\mathrm{B} / \mathrm{C}$ & $53 / 86$ & $51 / 88$ & $0.901^{\mathrm{b}}$ \\
\hline
\end{tabular}

Data are expressed as number or mean \pm standard deviation. B, hepatitis B virus; C, hepatitis C virus; HCC, hepatocellular carcinoma; ECOG PS, Eastern Cooperative Oncology Group Performance Status; AST, aspartate aminotransferase; ALT, alanine aminotransferase; AFP, $\alpha$-fetoprotein; DCP, des- $\gamma$-carboxy prothrombin; BCLC, Barcelona Clinic Liver Cancer. ${ }^{a}$ Unpaired t-test; ${ }^{b}$ Fisher's exact test.

Propensity score analysis. For reducing the bias in patient selection, a propensity score matching analysis was performed to examine causal relationships between initial dose of sorafenib ( 800 or $400 \mathrm{mg} / \mathrm{day})$ and clinical outcomes in a retrospective study other than a randomized controlled trial (RCT). Clinical variables entered into the propensity model were age, body weight, gender, Child-Pugh score and ECOG-PS, which indicated variables we frequently take into account in daily clinical practice when we decide initial dose of sorafenib (20). Subsequently, a one-to-one match between the standard-dose group $(800 \mathrm{mg} / \mathrm{day}$ of sorafenib) and the half-dose group $(400 \mathrm{mg} /$ day of sorafenib) was obtained by using the nearestneighbor matching method $(21,22)$.

\section{Results}

Baseline characteristics before propensity score matching. Baseline characteristics in the standard-dose group $(\mathrm{n}=183)$ and the half-dose group $(\mathrm{n}=254)$ prior to sorafenib therapy before propensity score matching are demonstrated in Table I. There were no relevant differences among two groups with respect to gender $(\mathrm{P}=0.104)$, HCC stage $(\mathrm{P}=0.168), \mathrm{BCLC}$ stage ( $\mathrm{P}=0.762)$, ECOG-PS $(\mathrm{P}=0.626)$, total bilirubin $(\mathrm{P}=0.230)$, aspartate aminotransferase $(\mathrm{P}=0.740), \alpha$-fetoprotein (AFP) $(\mathrm{P}=0.054)$ and des- $\gamma$-carboxy prothrombin $(\mathrm{P}=0.155)$, whereas in terms of age $(\mathrm{P}<0.001)$, height $(\mathrm{P}=0.020)$, body weight $(\mathrm{P}=0.012)$, cause of liver disease $(\mathrm{P}=0.001)$, Child-Pugh score $(\mathrm{P}=0.002)$, serum albumin $(\mathrm{P}=0.043)$, alanine aminotrans- ferase $(\mathrm{P}=0.019)$ and cholinesterase $(\mathrm{P}=0.010)$, significant differences were found in the two groups. The most frequently performed previous therapy for HCC was TACE in both groups.

Comparison of OS and PFS rates in the two groups before propensity score matching. The median follow-up periods after sorafenib therapy before propensity score matching were 6.9 months (range, $0.5-46.2$ months) in the standard-dose group and 7.9 months (range, $0.3-41.4$ months) in the half-dose group. The median OS intervals were 8.8 months [95\% confidence interval (CI), 6.9-10.7 months] in the standard-dose group and 9.4 months (95\% CI, 7.6-11.1 months) in the half-dose group ( $\mathrm{P}=0.913$ ) (Fig. 2). The median PFS intervals were 3.4 months (95\% CI, 3.0-3.7 months) in the standard-dose group and 3.3 months (95\% CI, 2.9-3.7 months) in the half-dose group $(\mathrm{P}=0.875)$ (Fig. 3).

Baseline characteristics, OS and PFS after propensity score matching. Baseline characteristics in the two groups prior to sorafenib therapy after propensity score matching (139 pairs) are shown in Table II. The median follow-up periods after propensity score matching were 7.1 months (range, 0.5-46.2 months) in the standard-dose group and 9.0 months (range, 0.7-41.4 months) in the half-dose group. In baseline characteristics, there were no relevant differences among two groups except for AFP-value ( $\mathrm{P}=0.043)$. The median OS intervals were 9.2 months (95\% CI, 7.3-11.0 months) in the 


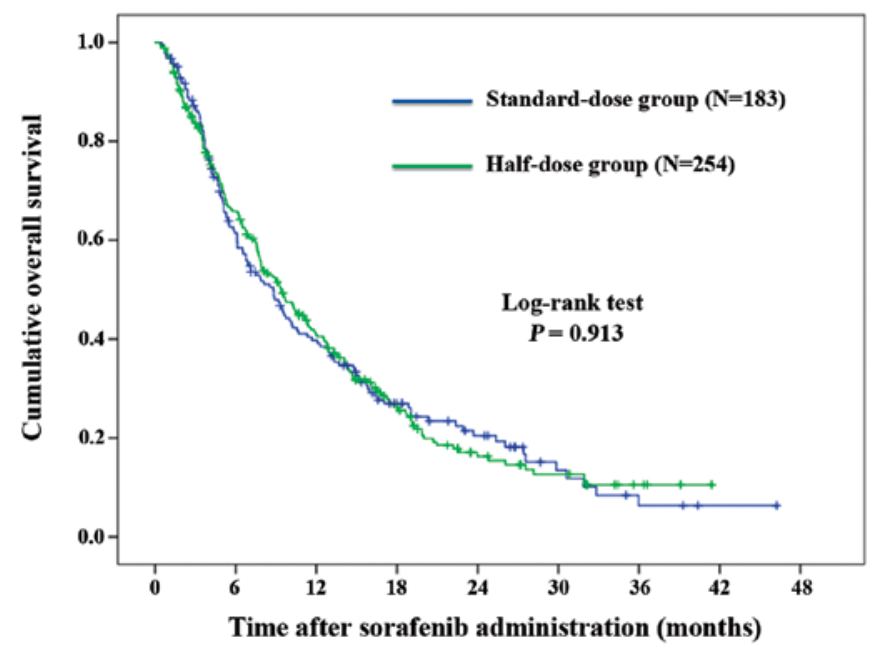

Figure 2. Cumulative overall survival (OS) in the standard-dose group $(n=183)$ and the half-dose group $(n=254)$ before propensity score matching. The median OS intervals were 8.8 months [95\% confidence interval (CI)], 6.9-10.7 months) in the standard-dose group and 9.4 months (95\% CI, 7.6-11.1 months) in the half-dose group ( $\mathrm{P}=0.913)$.

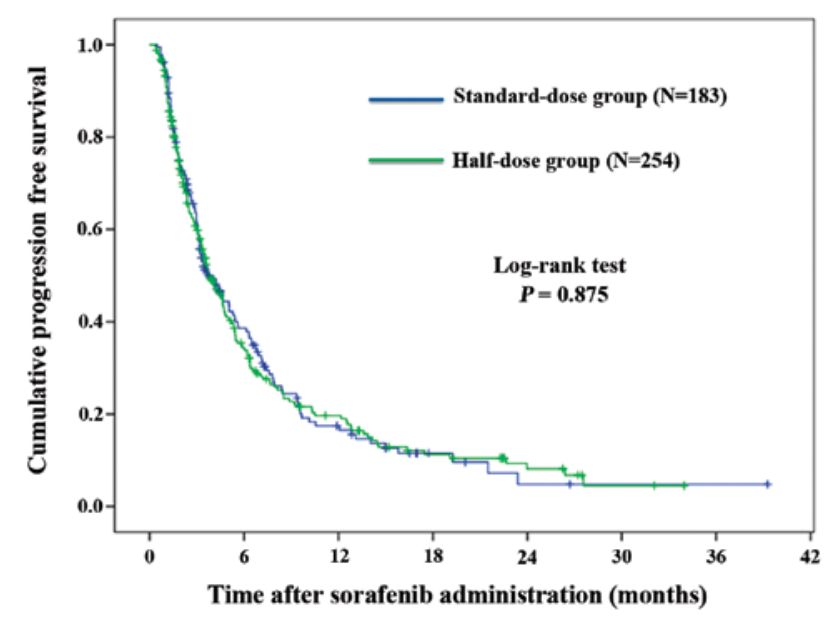

Figure 3. Cumulative progression-free survival (PFS) in the standard-dose group $(n=183)$ and the half-dose group $(n=254)$ before propensity score matching. The median PFS intervals were 3.4 months (95\% CI, 3.0-3.7 months) in the standard-dose group and 3.3 months (95\% CI, 2.9-3.7 months) in the half-dose group $(\mathrm{P}=0.875)$.

standard-dose group and 9.7 months (95\% CI, 7.8-11.7 months) in the half-dose group ( $\mathrm{P}=0.350)$ (Fig. 4). The median PFS intervals were 3.4 months (95\% CI, 2.6-4.2 months) in the standard-dose group and 3.2 months (95\% CI, 2.5-3.9 months) in the half-dose group ( $\mathrm{P}=0.729)$ (Fig. 5).

Treatment duration, treatment discontinuation rate and dose reduction or escalation rate in the two groups after propensity score matching. In the standard-dose group, the median treatment duration period of sorafenib after propensity score matching was 3.1 months (range, 0.1-39.3 months). Sorafenib treatment was discontinued in 127 patients $(91.4 \%)$ and sorafenib dose was reduced in 85 patients $(61.2 \%)$ the sorafenib dose was not escalated in any patient during the follow-up period. The mean administered sorafenib dose per day was $628 \mathrm{mg}$

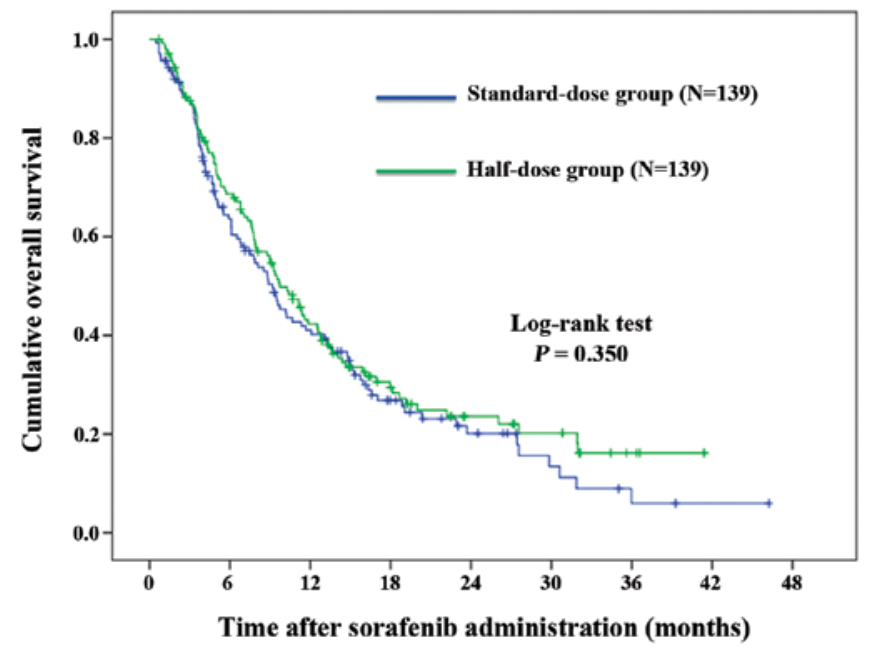

Figure 4. Cumulative overall survival (OS) in the standard-dose group $(\mathrm{n}=139)$ and the half-dose group $(\mathrm{n}=139)$ after propensity score matching. The median OS intervals were 9.2 months (95\% CI, 7.3-11.0 months) in the standard-dose group and 9.7 months (95\% CI, 7.8-11.7 months) in the half-dose group $(\mathrm{P}=0.350)$.

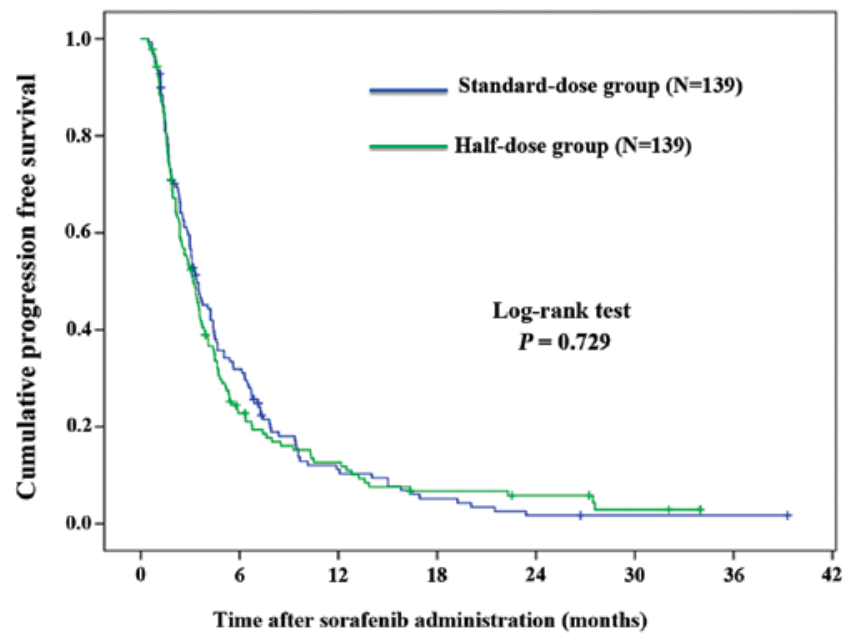

Figure 5. Cumulative progression-free survival (PFS) in the standarddose group $(n=139)$ and the half-dose group $(n=139)$ after propensity score matching. The median PFS intervals were 3.4 months $(95 \% \mathrm{CI}$, 2.6-4.2 months) in the standard-dose group and 3.2 months (95\% CI, 2.5-3.9 months) in the half-dose group $(\mathrm{P}=0.729)$.

(missing data for some patients). In the half-dose group, the median treatment duration period of sorafenib after propensity score matching was 3.9 months (range, 0.1-32.1 months). Sorafenib treatment was discontinued in 121 patients $(87.1 \%)$ and sorafenib dose was reduced in 44 patients $(31.7 \%)$ and sorafenib dose was escalated in 36 patients $(25.9 \%)$ during follow-up period. The mean administered sorafenib dose per day was $395 \mathrm{mg}$ (missing data for some patients). Most patients in the two groups discontinued sorafenib therapy due to progressive disease or sorafenib related SAEs.

Cause of death in the two groups after propensity score matching. During follow-up period, 101 patients (72.7\%) in the standard-dose group died. The causes of death in the standarddose group were HCC progression in 73 patients, liver failure 
Table III. Sorafenib related adverse events in the standard-dose group and the half-dose group after propensity score matching.

\begin{tabular}{|c|c|c|c|c|c|c|}
\hline & \multicolumn{2}{|c|}{ Standard-dose group $(\mathrm{N}=139)$} & \multicolumn{2}{|c|}{ Half-dose group $(\mathrm{N}=139)$} & \multicolumn{2}{|c|}{ P-value ${ }^{a}$} \\
\hline & $\begin{array}{c}\text { Any grade } \\
\text { n }(\%)\end{array}$ & $\begin{array}{c}\text { Grade } 3 \text { or more } \\
\mathrm{n}(\%)\end{array}$ & $\begin{array}{c}\text { Any grade } \\
\text { n }(\%)\end{array}$ & $\begin{array}{c}\text { Grade } 3 \text { or more } \\
\mathrm{n}(\%)\end{array}$ & Any grade & Grade 3 or more \\
\hline Overall & $127(91.4)$ & $37(26.6)$ & $127(91.4)$ & $33(23.7)$ & $>0.999$ & 0.580 \\
\hline HFSR & $77(55.4)$ & $8 \quad(5.8)$ & $62(44.6)$ & $7 \quad(5.0)$ & 0.092 & $>0.999$ \\
\hline Rash & $27(19.4)$ & $7 \quad(5.0)$ & $24(17.3)$ & $6(4.3)$ & 0.757 & $>0.999$ \\
\hline Diarrhea & $43(30.9)$ & $2(1.4)$ & $44(31.7)$ & $1(0.7)$ & $>0.999$ & $>0.999$ \\
\hline Hypertension & $26(18.7)$ & $2(1.4)$ & $19(13.7)$ & $3(2.2)$ & 0.328 & $>0.999$ \\
\hline Fatigue & $69(49.6)$ & $5 \quad(3.4)$ & $69(49.6)$ & $4(2.9)$ & $>0.999$ & $>0.999$ \\
\hline Liver injury & $60(43.2)$ & $15(10.8)$ & $61(43.9)$ & $16(11.5)$ & $>0.999$ & $>0.999$ \\
\hline Gastrointestinal bleeding & $9(6.5)$ & $3(2.2)$ & $5 \quad(3.4)$ & $0 \quad(0.0)$ & 0.206 & 0.247 \\
\hline Lung injury & $4 \quad(2.9)$ & $3(2.2)$ & $8 \quad(5.8)$ & $3(2.2)$ & 0.255 & $>0.999$ \\
\hline
\end{tabular}

HFSR, hand foot skin reaction. ${ }^{a}$ Fisher's exact test.

in 16, sorafenib related SAEs in 2 and miscellaneous causes in 10 . On the other hand, 98 patients $(70.5 \%)$ in the half-dose group died during follow-up period. The causes of death in the half-dose group were HCC progression in 81 patients, liver failure in 10 and miscellaneous causes in 7.

Best tumor response in the two groups after propensity score matching. In the standard-dose group, regarding the best tumor response, CR was obtained in 2, PR in 23, SD in 45, PD in 44 and not evaluated (NE) in 25. Thus, ORR was $18.0 \%(25 / 139)$ and DCR was $50.4 \%(70 / 139)$ in the standard-dose group. In the half-dose group, regarding best tumor response, CR was obtained in 2, PR in 18, SD in 54, PD in 45 and NE in 20. Thus, ORR was $14.4 \%$ (20/139) and DCR was 53.2\% (74/139) in the half-dose group. There was no relevant difference in the two groups in terms of best treatment efficacy (ORR: $\mathrm{P}=0.416$, DCR: $\mathrm{P}=0.719$ ).

Serious adverse events (SAEs) after propensity score matching. Any grade SAEs as defined by CTCAE were found in 127 patients $(91.4 \%)$ in the standard-dose group and 127 patients $(91.4 \%)$ in the half-dose group ( $\mathrm{P}>0.999)$. The most frequently observed SAE (any grade) was HFSR (55.4\%) in the standard-dose group and fatigue (49.6\%) in the half-dose group. On the other hand, grade 3 or more SAEs as defined by CTCAE were observed in 37 patients $(26.6 \%)$ in the standarddose group and 33 patients $(23.7 \%)$ in the half-dose group $(\mathrm{P}=0.580)$. The most frequently observed SAE (grade 3 or more) was liver injury $(10.8 \%)$ in the standard-dose group and liver injury (11.5\%) in the half-dose group (Table III).

Subgroup analyses according to BCLC stage and ChildPugh classification after propensity score matching. We also performed subgroup analyses according to BCLC stage and Child-Pugh classification after propensity score matching as these variables are well known prognostic factors in HCC patients.
In patients with BCLC-B HCC ( $\mathrm{n}=53$ in the standard-dose group and $n=51$ in the half-dose group), the median survival times (MSTs) (95\% CIs) were 13.3 months (9.1-17.6 months) in the standard-dose group and 14.7 months (10.6-18.7 months) in the half-dose group $(\mathrm{P}=0.522)$, whereas in patients with BCLC-C HCC ( $n=86$ in the standard-dose group and $n=88$ in the half-dose group), the MSTs (95\% CIs) were 6.5 months (4.1-8.9 months) in the standard-dose group and 7.8 months (5.8-9.8 months) in the half-dose group $(\mathrm{P}=0.418)$.

In patients with Child-Pugh A HCC ( $n=118$ in the standard-dose group and $n=125$ in the half-dose group), the MSTs (95\% CIs) were 10.7 months (6.9-14.4 months) in the standarddose group and 10.4 months (8.3-12.5 months) in the half-dose group $(\mathrm{P}=0.910)$, while in patients with Child-Pugh B HCC $(n=21$ in the standard-dose group and $n=14$ in the half-dose group), the MSTs (95\% CIs) were 4.2 months (3.0-5.3 months) in the standard-dose group and 5.1 months (2.4-7.9 months) in the half-dose group $(\mathrm{P}=0.058)$, indicating that there was trend for better survival in HCC patients with Child-Pugh B treated with initial sorafenib dose of $400 \mathrm{mg} / \mathrm{day}$.

\section{Discussion}

There have been few studies comparing the clinical outcome and safety between different initial doses of sorafenib administered to HCC patients $(10,13)$. HCC patients enrolled in RCTs do not necessarily represent the field practice owing to the absence of potential confounding factors such as comorbid diseases. In addition, the results of clinical studies of sorafenib therapy for HCC performed in Japan revealed that $>80 \%$ of enrolled patients treated with initial standarddose sorafenib (800 mg/day) required dose reduction (23). Thus, there is urgent need for investigating the usefulness of initial reduced dose of sorafenib therapy in HCC patients in field practice. Hence, we aimed to conduct this multicenter comparative study using propensity score matching analysis for reducing selection biases. The major strengths of the 
current analyses were the large sample size $(n=278$ after propensity score matching), the consecutive enrollment of Japanese HCC patients with broad eligibility criteria reflecting the diversity and complexity of our field practice for HCC and the involvement of 14 centers with well-referenced expertise in $\mathrm{HCC}$ diagnosis and treatment for HCC.

In the present study, there were no significant differences in terms of OS, PFS, best treatment response and SAEs in the standard-dose and the half-dose groups after propensity score matching or in the subgroup analyses after propensity score matching, no significant difference was observed in the two groups. Our results suggest that initial half-dose sorafenib therapy for HCC can be a treatment option for some patients. While the interim analysis in the GIDEON study demonstrated a trend toward more evident clinical benefits for initial standard-dose sorafenib as compared with the initial half-dose sorafenib and patients who initiated standard-dose sorafenib tended to discontinue treatment later than patients who initiated half-dose sorafenib (12.3 vs. 9.7 weeks) and present a longer OS (9.3 vs. 7.1 months) and time to progression (4.5 vs. 3.6 months) $(10,11,24)$. On the other hand, a recent comparative study reported from Japan showed that using propensity score matching, HCC patients treated with the initial half-dose sorafenib therapy $(n=58)$ led to a comparable survival benefit compared with those treated with the standard-dose sorafenib therapy $(n=58)$, which are in line with our present study results (13). Furthermore, the results in the SOFIA study confirmed the safety and treatment effectiveness of sorafenib in a real-life clinical setting even with a reduced dose (9). The authors in the SOFIA study demonstrated that the median OS was 10.5 months in the overall cohort $(n=296)$ [8.4 months in BCLC-C vs. 20.6 months in BCLC-B patients $(\mathrm{P}<0.0001)$ ], and 21.6 months in the 77 patients treated for $\geq 70 \%$ of the time with a half-dose sorafenib vs. 9.6 months in the 219 patients with standard-dose sorafenib or half-dose sorafenib $<70 \%$ treatment period $(\mathrm{P}=0.0006)(9)$. The discrepancies for these study results may be attributed to different baseline characteristics such as race, age, body weight, extension of liver disease and background liver disease in these studies $(1,2,4,5,25,26)$. For instance, the mean age in our current analysis was $\sim 70$ years, while that in the GIDEON study was 62 years (several Japanese HCC patients were included in the GIDEON study), which is 8 years younger than our study population $(10,11,24)$. Further well defined comparative studies will thus be needed in the future to confirm these results.

It is of note that in our subgroups analyses in patients with Child-Pugh $B(n=21$ in the standard-dose group and $n=14$ in the half-dose group), the median OS tended to be longer in the half-dose group than the standard-dose group (5.1 vs. 4.2 months, $\mathrm{P}=0.058$ ) and the median duration of sorafenib therapy was 3.3 months in the half-dose group and 1.6 months in the standard-dose group (data not shown). Available evidence suggests that the safety profile of sorafenib therapy is comparable in HCC patients with Child-Pugh A and B, however, half-dose sorafenib therapy can be recommended in HCC patients with poor liver function of Child-Pugh B for avoiding treatment discontinuation due to SAEs considering our results of subgroup analyses $(10,11)$.

In our analyses of sorafenib related SAEs, grade 3 or more sorafenib related SAEs in the standard-dose and half-dose groups were found in 37 patients $(26.6 \%)$ and 33 patients $(23.7 \%)$, whereas in the GIDEON study, grade 3 or more sorafenib related SAEs in the standard-dose and half-dose groups were found in 274 out of 1,161 patients (24\%) and 84 out of 347 patients (24\%), which are similar to our results (11). On the other hand, in the present study, sorafenib treatment was discontinued in 127 patients $(91.4 \%)$ in the standard-dose group and 121 patients $(87.1 \%)$ in the half-dose group, which are higher than the discontinuation rate of sorafenib in the SHARP study [226 out of 297 patients (sorafenib arm), 76.9\%] (7). The higher prevalence of patients with Child-Pugh B in this study $(15.1 \%$ in the standard-dose group and $10.1 \%$ in the half-dose group) compared with that in the SHARP study (5\%, sorafenib arm) could in part account for high rates of treatment discontinuation of our present study (7).

In our propensity score matching, clinical variables including age, body weight, gender, Child-Pugh score and ECOG-PS were entered. All these variables are key factors when deciding initial dose of sorafenib in field practice. After propensity score matching, the distributions of all confounding factors except for AFP-value in the two groups were well balanced for statistical analyses and we believe that this difference in the two groups after propensity score matching did not affect for interpreting our study results.

Our study included several limitations. Firstly, this is a retrospective study although propensity score matching analysis for reducing selection biases was performed. Secondly, various therapies for HCC were performed after discontinuation of sorafenib, potentially leading to bias for evaluating OS. Thirdly, in this study, dose adjustment of sorafenib during treatment was decided mainly based on the decision of each attending physician, also leading to bias. Fourthly, our study cohort included only Japanese HCC patients, who in general had lower body weight than populations in Western countries $(25,26)$. Hence, caution should be exercised for interpreting our results. However, our study results demonstrated that HCC patients treated with half-dose sorafenib had comparable clinical outcomes compared with those treated with standarddose sorafenib. In conclusion, reduced initial dose of sorafenib may not affect clinical outcomes for patients with unresectable HCC especially in Japanese HCC patients with relatively lower body weight.

\section{Acknowledgements}

The authors would like to thank all the staff in Japanese Red Cross Liver Study Group for their valuable support. This study was supported by Health and Labour Sciences Research Grant.

\section{References}

1. El-Serag HB: Hepatocellular carcinoma. N Engl J Med 365: 1118-1127, 2011.

2. El-Serag HB: Epidemiology of viral hepatitis and hepatocellular carcinoma. Gastroenterology 142: 1264-1273, 2012.

3. Lencioni R: Loco-regional treatment of hepatocellular carcinoma. Hepatology 52: 762-773, 2010.

4. Nishikawa $\mathrm{H}$ and Osaki Y: Non-B, non-C hepatocellular carcinoma (Review). Int J Oncol 43: 1333-1342, 2013.

5. Osaki Y and Nishikawa H: Treatment for hepatocellular carcinoma in Japan over the last three decades: our experience and literature review. Hepatol Res: Jun 26, 2014 (Epub ahead of print). doi: 10.1111/hepr.12378. 
6. Nishikawa H, Arimoto A, Wakasa T, Kita R, Kimura T and Osaki Y: Effect of transcatheter arterial chemoembolization prior to surgical resection for hepatocellular carcinoma. Int J Oncol 42: 151-160, 2013.

7. Llovet JM, Ricci S, Mazzaferro V, Hilgard P, Gane E, Blanc JF, de Oliveira AC, Santoro A, Raoul JL, Forner A, Schwartz M, Porta C, Zeuzem S, Bolondi L, Greten TF, Galle PR, Seitz JF, Borbath I, Häussinger D, Giannaris T, Shan M, Moscovici M, Voliotis D and Bruix J; SHARP Investigators Study Group: Sorafenib in advanced hepatocellular carcinoma. N Engl J Med 359: 378-390, 2008

8. Cheng AL, Kang YK, Chen Z, Tsao CJ, Qin S, Kim JS, Luo R, Feng J, Ye S, Yang TS, Xu J, Sun Y, Liang H, Liu J, Wang J, Tak WY, Pan H, Burock K, Zou J, Voliotis D and Guan Z: Efficacy and safety of sorafenib in patients in the Asia-Pacific region with advanced hepatocellular carcinoma: a phase III randomised, double-blind, placebo-controlled trial. Lancet Oncol 10: 25-34, 2009.

9. Iavarone M, Cabibbo G, Piscaglia F, Zavaglia C, Grieco A, Villa E, Cammà C and Colombo M; SOFIA (SOraFenib Italian Assessment) study group: Field-practice study of sorafenib therapy for hepatocellular carcinoma: a prospective multicenter study in Italy. Hepatology 54: 2055-2063, 2011.

10. Lencioni R, Kudo M, Ye SL, Bronowicki JP, Chen XP, Dagher L, Furuse J, Geschwind JF, Ladrón de Guevara L, Papandreou C, Sanyal AJ, Takayama T, Yoon SK, Nakajima K, Cihon F, Heldner S and Marrero JA: First interim analysis of the GIDEON (Global Investigation of therapeutic decisions in hepatocellular carcinoma and of its treatment with sorafeNib) non-interventional study. Int J Clin Pract 66: 675-683, 2012.

11. Lencioni R, Kudo M, Ye SL, Bronowicki JP, Chen XP, Dagher L, Furuse J, Geschwind JF, de Guevara LL, Papandreou C, Takayama T, Yoon SK, Nakajima K, Lehr R, Heldner S and Sanyal AJ: GIDEON (Global Investigation of therapeutic DEcisions in hepatocellular carcinoma and Of its treatment with sorafeNib): second interim analysis. Int J Clin Pract 68: 609-617, 2014.

12. Di Marco V, De Vita F, Koskinas J, Semela D, Toniutto P and Verslype C: Sorafenib: from literature to clinical practice. Ann Oncol 24 (Suppl 2): ii30-ii37, 2013.

13. Morimoto M, Numata K, Kondo M, Kobayashi S, Ohkawa S, Hidaka H, Nakazawa T, Okuwaki Y, Okuse C, Matsunaga K, Suzuki M, Morita S, Taguri M and Tanaka K: Field practice study of half-dose sorafenib treatment on safety and efficacy for hepatocellular carcinoma: A propensity score analysis Hepatol Res: May 6, 2014 (Epub ahead of print). doi: 10.1111/ hepr.12354

14. Takeda H, Nishikawa H, Osaki Y, Tsuchiya K, Joko K, Ogawa C, Taniguchi H, Orito E, Uchida Y and Izumi N; Japanese Red Cross Liver Study Group: Clinical features associated with radiological response to sorafenib in unresectable hepatocellular carcinoma: a large multicenter study in Japan. Liver Int: May 16, 2014 (Epub ahead of print). doi: 10.1111/liv.12591.

15. Nishikawa H, Takeda H, Tsuchiya K, Joko K, Ogawa C, Taniguchi H, Orito E, Uchida Y, Osaki Y and Izumi N; Japanese Red Cross Liver Study Group: Sorafenib therapy for BCLC stage $\mathrm{B} / \mathrm{C}$ hepatocellular carcinoma; clinical outcome and safety in aged patients: a multicenter study in Japan. J Cancer 5: 499-509, 2014.
16. Cheng AL, Amarapurkar D, Chao Y, Chen PJ, Geschwind JF, Goh KL, Han KH, Kudo M, Lee HC, Lee RC, Lesmana LA, Lim HY, Paik SW, Poon RT, Tan CK, Tanwandee T, Teng G and Park JW: Re-evaluating transarterial chemoembolization for the treatment of hepatocellular carcinoma: consensus recommendations and review by an International Expert Panel. Liver Int 34: 174-183, 2014.

17. European Association For The Study Of The Liver; European Organisation For Research And Treatment Of Cancer: EASL-EORTC clinical practice guidelines: management of hepatocellular carcinoma. J Hepatol 56: 908-943, 2012.

18. Lencioni R and Llovet JM: Modified RECIST (mRECIST) assessment for hepatocellular carcinoma. Semin Liver Dis 30: 52-60, 2010.

19. Salvaggio G, Furlan A, Agnello F, Cabibbo G, Marin D, Giannitrapani L, Genco C, Midiri M, Lagalla R and Brancatelli G: Hepatocellular carcinoma enhancement on contrast-enhanced CT and MR imaging: response assessment after treatment with sorafenib: preliminary results. Radiol Med 119: 215-221, 2014.

20. Sacco R, Bargellini I, Ginanni B, Bertini M, Faggioni L, Federici G, Romano A, Bertoni M, Metrangolo S, Altomare E, Parisi G, Tumino E, Scaramuzzino A, Bresci G and Bartolozzi C: Long-term results of sorafenib in advanced-stage hepatocellular carcinoma: what can we learn from routine clinical practice? Expert Rev Anticancer Ther 12: 869-875, 2012.

21. D'Agostino RB Jr: Propensity score methods for bias reduction in the comparison of a treatment to a non-randomized control group. Stat Med 17: 2265-2281, 1998.

22. Seung KB, Park DW, Kim YH, Lee SW, Lee CW, Hong MK, Park SW, Yun SC, Gwon HC, Jeong MH, Jang Y, Kim HS, Kim PJ, Seong IW, Park HS, Ahn T, Chae IH, Tahk SJ, Chung WS and Park SJ: Stents versus coronary-artery bypass grafting for left main coronary artery disease. N Engl J Med 358: 1781-1792, 2008.

23. Ogasawara S, Kanai F, Obi S, Sato S, Yamaguchi T, Azemoto R, Mizumoto H, Koushima Y, Morimoto N, Hirata N, Toriyabe T, Shinozaki Y, Ooka Y, Mikata R, Chiba T, Okabe S, Imazeki F, Yoshikawa M and Yokosuka O: Safety and tolerance of sorafenib in Japanese patients with advanced hepatocellular carcinoma. Hepatol Int 5: 850-856, 2011.

24. Marrero J, Venook A and Kudo M: Second interim analysis of GIDEON (Global Investigation of therapeutic DEcisions in unresectable hepatocellular carcinoma and Of its treatment with sorafeNib): subgroup analysis by initial sorafenib dose. Hepatology 54: n2119, 2011.

25. McCurry J: Japan battles with obesity. Lancet 369: 451-452, 2007.

26. Examination Committee of Criteria for 'Obesity Disease' in Japan; Japan Society for the Study of Obesity: New criteria for 'obesity disease' in Japan. Circ J 66: 987-992, 2002. 